Research Article

\title{
Supply Chain Decision Model Based on Blockchain: A Case Study of Fresh Food E-Commerce Supply Chain Performance Improvement
}

\author{
Zi-Yu Liu $(D)$ and Peng-Tao Guo iD \\ School of Economics and Management, Hebei University of Science and Technology, Shijiazhuang, Hebei 050018, China \\ Correspondence should be addressed to Zi-Yu Liu; purpleyuliu@163.com
}

Received 13 June 2021; Revised 24 June 2021; Accepted 3 July 2021; Published 13 July 2021

Academic Editor: Daqing Gong

Copyright (c) $2021 \mathrm{Zi}-\mathrm{Yu}$ Liu and Peng-Tao Guo. This is an open access article distributed under the Creative Commons Attribution License, which permits unrestricted use, distribution, and reproduction in any medium, provided the original work is properly cited.

\begin{abstract}
Aiming at the problems in the performance improvement of the fresh food e-commerce supply chain, in this article, the author defines the constraints on the performance improvement of the fresh food e-commerce supply chain and determines the demand to improve performance in quality and safety, logistics traceability, resource integration, and benefit distribution. In addition, this article conducts coupling analysis on the characteristics of blockchain technology, such as intelligent contract, information sharing, traceability, and performance improvement requirements. The author embeds the alliance chain in the fresh food e-commerce supply chain and designs the blockchain information platform model from four levels: application layer, contract layer, network layer, and data layer, with the purpose of optimizing and improving the performance of the fresh food e-commerce supply chain. What is more, the Stackelberg game model between the supplier and the e-commerce platform was constructed to study and compare the profit changes of the fresh food e-commerce supply chain before and after the application of the blockchain information platform. Finally, the result of Stackelberg game is numerically simulated by Matlab software. The results show that blockchain technology is a booster for the development of the fresh food e-commerce supply chain to a higher stage of the whole industry chain management, coordination, and integration. Investing in the blockchain system within a certain budget range can not only improve the reliability of products, but also improve the performance of each main component of the fresh food e-commerce supply chain, as well as the overall performance. Results can offer a theoretical guidance for producer and retailer in investing in BIP, pricing decision, and supply chain coordination after applying BIP.
\end{abstract}

\section{Introduction}

Fresh food e-commerce, also known as fresh agricultural product e-commerce platform, is an information technologybased e-commerce platform that retails fresh products such as vegetables, meat, and aquatic products. In the meantime, there are also other business formats and corporate activities [1]. According to the data in the "2020 China Agricultural Products E-Commerce Development Report," China's fresh food e-commerce transactions in 2017-2019 were 141.8 billion yuan, 215.8 billion yuan, and 350.6 billion yuan. With the rapid increase in transaction volume, on the one hand, fresh food e-commerce companies have "increased" accordingly, and on the other, there is a "development bottleneck" in fresh food e-commerce. During the 15 years of development of fresh food e-commerce, more than 20 fresh food e-commerce companies will lose money and close down every year. In 2019, 27 stores, such as Yiguo Shengxian, Mini Shengxian, and Xian laiduo, closed down due to losses. Fresh food e-commerce is rapidly expanding, but with poor supply chain collaboration, lack of integrity, long industrial chain, and large losses. And it did not form a "network chain" dominated by four chains, including industrial chain, supply chain, value chain, and blockchain, which in turn affects the overall performance of business operations and the fresh food supply chain. Therefore, the performance improvement and healthy development of fresh food e-commerce has become an urgent problem to be solved. 
At present, the core competitiveness of price discounts and distribution services is not enough to support the longterm development of fresh food e-commerce. In the "Outline of Digital Village Development" in 2019, the role of blockchain in promoting e-commerce of fresh agricultural products was emphasized. The Central Document No. 1 in 2020 focuses on the issues of "agriculture, rural areas, and farmers" and emphasizes the application of blockchain in the agricultural field [2]. Just as SARS promoted the development of Jack Mas e-commerce in 2003, the 2019-nCoV epidemic is also an opportunity for fresh food e-commerce companies to enter a new stage of digital development. The core of competition for fresh food e-commerce companies lies in the strength of the supply chain behind them. Blockchain technology can solve the internal and external factors affecting performance such as the information asymmetry of members in the fresh food e-commerce supply chain, product quality and safety, and logistics service quality. There are multiple couplings between the information sharing, smart contracts, and traceability characteristics of blockchain technology and the overall performance improvement needs of the fresh food e-commerce supply chain. This is of great significance to the income increase and healthy development of fresh food e-commerce.

In view of this, this article starts from the application of blockchain technology to the fresh food e-commerce supply chain to improve overall performance. First of all, by analyzing the existing relevant literature on the fresh food e-commerce supply chain and blockchain, the coupling between the characteristics of blockchain technology and the demand characteristics of fresh food e-commerce performance improvement was summarized. In addition, the author designed a fresh food supply chain information platform based on the consortium blockchain to realize the demand to improve performance of each member of the fresh food e-commerce supply chain, thereby promoting the performance improvement of the fresh food e-commerce supply chain. Then, the paper uses game theory to study the performance improvement of the fresh food supply chain in the context of blockchain, explores the application of blockchain technology in the fresh food supply chain, and puts forward practical and feasible performance improvement suggestions for fresh food e-commerce companies.

\section{Literature Review}

2.1. Research on Restrictive Factors of Fresh Food E-Commerce Supply Chain Performance Improvement. Supply chain performance is the result of the execution of supply chain strategy [3]. The ultimate goal of the supply chain is to increase the supply chain performance; therefore, improving the overall performance of supply chain is the purpose of performance optimization in this paper. The overall profit improvement of the supply chain is the purpose for this article to analyze the performance optimization. Different from the traditional agricultural product supply chain, the fresh food e-commerce supply chain is based on modern Internet information technology, with fresh food e-commerce companies as the leading enterprises, connecting the various components, including a series of links such as production, procurement, service, sales, and distribution [4]. There are not many studies on the factors affecting the performance of the fresh food e-commerce supply chain at home and abroad. They can be roughly divided into two types: theoretical researches that propose performance optimization countermeasures to the development status and empirical researches that explore the impact of various factors on performance through evaluation models.

The basic theoretical researches include the following: Van der Vorst emphasized that fresh products have special characteristics such as perishability, short shelf life, seasonal production, variability in quality and quantity, long production throughput time, and special transportation requirements [5]. With fresh food e-commerce as the last "blue ocean" in the e-commerce field, the driving factors that affect its supply chain performance include not only external factors such as industry characteristics, technologies, and customers, but also internal factors such as processes, partnerships, and supply chain strategies [3]; Joby George and Pillai pointed out that the main factors affecting supply chain performance are supply chain structure, inventory control, policies, information sharing, customer demand, forecasting methods, preparation time, and review period length [6]; Zan et al. conducted a comprehensive review of the development history of domestic fresh food e-commerce, put forward hindering factors such as the difficulty of building fresh food e-commerce brands, high operating costs, vicious competition on the supply side, and friction with the traditional market, and suggested refining procurement standards and improving the degree of informatization, etc. [7].

Empirical researches on the performance evaluation and optimization of the fresh food supply chain include the following: based on five performance criteria, Moazzam et al. summarized the SCOR model suitable for the performance and risk analysis of the fresh agricultural product supply chain by reviewing existing performance evaluation systems and frameworks. Environmental sustainability, food quality, flexibility, value at risk, and other indicators were added in the selected reliability, responsiveness, agility, cost, and asset attributes, and through the case of New Zealand dairy products, the superiority of the analysis framework was verified through [8]; in the three-level fresh agricultural products supply chain, Odongo et al. used trust, commitments, information sharing, coercive force, noncoercive force, dependence, and conflict to represent the structure of supply chain relationship quality and explored the influence of supply chain relationship quality on supply chain performance. It was found that, in the upstream of fresh agricultural products, trust, commitment, coercive force, noncoercive force, dependence, and conflict were the most important attributes; in the downstream core enterprises, trust and conflict were the most important attributes, and customers believed that trust, dependence, and coercive force were the important attributes [9]; Yadav et al. developed a data-driven agricultural supply chain performance 
measurement framework based on the Internet of Things and added sustainability indicators to provide references for improving agricultural supply chain performance [10]; Yadav and Garg shared information at all levels of the agricultural product supply chain in real time through cloud-based information technology systems, solved the asymmetry of power distribution within the fresh supply chain, and improved supply chain fairness and performance [11]; Kaipia et al. studied how to use information sharing to reduce waste and to improve sustainable performance in the three fresh food supply chains of milk, fresh fish, and poultry [12]; Yeh et al. studied how the integration of the supply chain of small- and medium-sized enterprises affects the relationship stability of the supply chain, thereby affecting the supply chain performance. It is concluded that internal integration, supplier integration, and customer integration have positive impacts on relationship stability, relationship stability has a positive impact on supplier performance, and environmental uncertainty only moderates the impact of internal integration on relationship stability [13].

\subsection{Research on the Application of Blockchain Technology in} the Fresh Food E-Commerce Supply Chain. The characteristics of blockchain technology are decentralization, openness and transparency, incorrigibility of data, security and credibility, anonymity, traceability, etc., so it is widely used in the fields of finance, education, medical care, and supply chain management. As the underlying technology of Bitcoin, blockchain is essentially a chained data structure that links data blocks in chronological orders, a decentralized distributed database, or electronic account book that is tamperresistant, secure, and transparent protected by cryptography [14]. The data block is mainly composed of the block headers and the block bodies. The block headers include the version number, the hash value of the block, the hash value of the previous block, the random number of the solution of the previous blocks consensus process, the timestamp, and the Merkle root node. The block bodies include the current transaction records and all previous transaction records that have been successfully verified [15].

The research on the application of blockchain technology in the supply chain of fresh agricultural products is divided into four aspects.

(1) The researches on the safety traceability systems of fresh products are the fields that currently combine the most blockchain with the fresh supply chain. Zhou and Zheng built a four-level system framework (physical layer, core layer, data layer, and application layer) with e-commerce platform as the core for the current management of the whole industry chain after the food supervision reform and analyzed the traceability operation process [16]; Salah et al. proposed a tracking and tracing technology for soybeans in the agricultural supply chain based on the Ethereum blockchain, using smart contracts to control and manage the transactions and interactions of all participants [17]; Salah et al. propose an approach that leverages the Ethereum blockchain and smart contracts efficiently perform business transactions for soybean tracking and traceability across the agricultural supply chain [17]; Alzahrani proposed a decentralized anticounterfeiting supply chain using NFC and blockchain technology [18].

(2) In the logistics circulation of fresh agricultural products supply chain, the strict requirements for logistics efficiency in the fresh food e-commerce supply chain is another research hotspot. Li designed the three-tier blockchain deployment of the initial layer, the middle layer, and the consumer layer in response to the low circulation efficiency, low timeliness controllability, and high circulation costs of fresh agricultural products in China. Finally, the feasibility of the scheme was verified through empirical research and simulation [19]; Park explored the influencing factors of the application of blockchain technology in the logistics industry and pointed out that blockchain technology makes supply chain logistics more efficient and more competitive [20]. Based on the cold chain logistics of rural agricultural products, Mei aimed at the cold chain logistics of rural agricultural products, based on blockchain technology to construct a cold chain logistics model that encompasses the whole process from origin to sales [21].

(3) applications in supply chain finance, Tang and Zhuang constructed a blockchain debt-to-credit platform based on blockchain technology for supply chain financial credit problems and compared the advantages and disadvantages of the newsboy model with the traditional model; finally they verified the advantages of the blockchain debt-to-credit platform through numerical analysis [22]; Gauthier systematically studied the factoring business model based on blockchain smart contract technology and proposed a supply chain "intelligent factoring" system with simple design and operation, low operating costs, and convenient financing advantages [23].

(4) In the aspect of fresh food supply chain performance, most of them are empirical studies on various efficiency improvements brought by the addition of blockchain into the supply chain. Nandi et al. pointed out that the current work of supply chain system supporting blockchain technology focused more on improving the ability of the business level (information sharing and coordination) than the ability of the strategic level integration and collaboration capabilities, such as compliance and quality improvement, process improvement, flexibility, reduced costs, and reduced process time, so as to improve its supply chain performance [24]; Nandi et al. aimed to explore how firms' efforts to integrate blockchain technology (BCT) into their supply chain systems and activities enable certain supply chain capabilities and, consequently, improve their supply chain performance [24]. Hald and Kinra showed the 
important and complex Janus-faced implications of embedding BCT in supply chains and demonstrated how organizational theory can be applied to explore the relationship between blockchain and SCM [25]. Combined with the above literature, it is found that the application of blockchain technology in the fresh food e-commerce supply chain has a good foundation. However, most of the research results of blockchain are in the stage of improvement and the implementation is not strong.

By conducting a comprehensive analysis on the above literature, we found that (1) combining the industry characteristics of fresh food e-commerce and existing research literature of supply chain performance, according to external factors, such as industry characteristics, technology, and customers, and internal factors such as processes, partnerships, and supply chain strategies, it can be summarized that the constraints on the performance improvement of the fresh food supply chain studied in this article include food quality and safety issues, supply chain partnerships, supply chain collaboration and integration, customer experience, information technology, and other internal and external factors. (2) There are multiple couplings between the technical characteristics of the blockchain and the demand for performance improvement of the fresh food e-commerce supply chain. The partial application of blockchain in the fresh food e-commerce supply chain has achieved some results. For example, the quality and safety traceability system, the logistics circulation system, and the financing credit system have been optimized, but most of the results are in the stage of improvement and there are few practical applications. (3) Some domestic and foreign scholars have conducted empirical research on blockchain technology and the performance of fresh food e-commerce supply chain, which has laid a theoretical foundation for this article based on blockchain technology to integrate and coordinate the entire chain, improve the overall performance of the fresh food e-commerce supply chain, and increase the supply chain surplus.

In view of this, this article couples the technical characteristics of the blockchain with the performance constraints of the fresh food e-commerce supply chain, designs a blockchain-based fresh food e-commerce supply chain optimization model, integrates the resources of each node, strengthens information sharing, and promotes the integration and coordination of the supply chain to form a green and sustainable community of integrated fresh food e-commerce supply chain bearing the risk and sharing the profit, so as to improve overall performance. Then, this article uses game theory to verify whether the characteristics of the blockchain technology can promote the performance improvement of the fresh food e-commerce supply chain, explores the supply chain performance changes before and after the application of blockchain technology in the fresh food supply chain, provides guarantee for the implementation of blockchain technology, and puts forward suggestions for the development of fresh food e-commerce.

\section{Optimization Model of Fresh Food E-Commerce Supply Chain Based on Alliance Chain}

3.1. Blockchain Technology and Performance Optimization Demand Coupling. The performance of fresh food e-commerce supply chain is affected by multiple factors. After analyzing the literature, we found that fresh food quality and safety issues, partnerships in the supply chain, supply chain collaboration and integration, customer experience, and information technology and other internal and external factors affect the improvement of fresh food e-commerce supply chain performance. Based on the external driving factors, i.e., customers, and internal driving factors, i.e., partners, cost, and information, we select quality and safety, logistics traceability, relationship quality, resource integration and benefit distribution, and other factors that affect the performance of fresh e-commerce supply chain and analyze the coupling with the technical characteristics of blockchain. The customers in the fresh food e-commerce supply chain are the source of profit in the fresh food supply chain, and at the same time, they also represent the market trend. Product quality, service flexibility, and customer experience are the main influencing factors for performance. The quality of the relationship with the partner has a significant impact on performance. A good partnership in the supply chain is a win-win cooperation, and it has the advantages of reducing costs, increasing profits, forecasting accuracy, and controlling inventory. It can maintain the stability at the supply side and ensure the sustainability of the supply chain. Cost is an important input in the fresh food supply chain. The circulation cost and operating cost of fresh products, as well as the loss, are all important factors that affect performance. Information sharing is the key competitiveness of fresh food e-commerce supply chain. Sharing information can reduce the uncertainty of the fresh food supply chain, increase agility, reduce information asymmetry, increase trust, and improve the efficiency and performance of the fresh food e-commerce supply chain.

According to the restrictions on participating nodes, blockchains can be roughly divided into three types: public chain, similar to Bitcoin blockchain, where everyone can participate in checking transactions, obtaining consensus, and anonymous verification; private chain, which is formulated according to the rules of private organizations and requires organization authorization for reading and writing and consensus; the alliance chain, similar to China Ledge and Hyperledger, which formulates read and write permissions and obtains a consensus process according to the rules of the alliance. The data in the chain can be public or private and is limited to alliance members. 
At present, the consortium blockchain is suitable for applying in the fresh food e-commerce supply chain. Blockchain technology is decentralized (all network nodes have equal rights and obligations and share and maintain databases together); the data cannot be tampered with (asymmetric encryption and consensus mechanism ensure the safety of data and cannot be tampered with, and $51 \%$ of nodes need to be controlled to forge data). It is safe and trustworthy (the technologies such as hash algorithm, electronic signature, and digital certificate in cryptography are adopted to ensure the data security of block transaction). It also has the characteristics of smart contract and traceability. There are multiple couplings between the performance optimization requirements of the fresh food e-commerce supply chain (quality safety, logistics traceability, relationship quality, resource integration, and benefit distribution) and the characteristics of the blockchain technology (smart contracts, information sharing, data that cannot be tampered with, traceability, and decentralization), as shown in Figure 1. The application of blockchain technology to the fresh food e-commerce supply chain can weaken information asymmetry, improve the fairness between supply chain members, enhance their collaboration, and improve supply chain stability. The integration of suppliers, customers, and other node resources helps fresh food e-commerce to form an integration and enhance competitiveness. The sharing of information reduces the uncertainty of the fresh food e-commerce supply chain, enhances customer loyalty, forms the standard of branding, and promotes the integration of the fresh food e-commerce supply chain. In this way, the overall performance of fresh food e-commerce has been improved.

\subsection{Constructing an Optimization Model for the Blockchain} Information Platform of the Fresh Food E-Commerce Supply Chain. In order to solve the problems that hinder performance improvement in the fresh food e-commerce supply chain and promote the overall performance improvement and sustainable development of the fresh food e-commerce supply chain, we build a blockchain information platform with the help of blockchain technology to help the fresh food e-commerce supply chain move towards a standardized and integrated high-end model.

The consortium blockchain is a blockchain jointly organized and managed by multiple entities, institutions, or enterprises. The accounting node is preselected in advance, and the generation of new blockchain is jointly verified by the preselected nodes. Although it abandons complete decentralization, it is more in line with the needs of the industry. Based on the Byzantine consensus algorithm, the system records transactions quickly. Only when each member of the group agrees together can they establish their own guidelines of the system. In the field of fresh food e-commerce supply chain, the blockchain system is composed of five levels of data, network, consensus, contract, and application. We only design the basic architecture of the blockchain information platform in view of four levels (application layer, contract layer, network layer, and data layer) (see Figure 2). The application layer is a kind of encapsulation at the end of the client, which is for users to trace and check, supply chain members (manufacturers, logistics providers, fresh food e-commerce, etc.) to manage and query information, and regulatory authorities to inspect. The contract layer is a variety of smart contracts of enterprises, policies, and laws and automatic execution contracts such as script codes and algorithms, as well as programmable foundations. In the network layer, the P2P network structure is the basis of distributed storage. It also has a transmission mechanism to broadcast new transaction data and blocks to the whole network and the verification mechanism implemented according to the predetermined agreement. The data block at the data layer is mainly composed of the block head and the block body. The block head includes the version number, the hash value of the block, the hash value of the previous block, the random number of the solution of the previous blocks consensus process, the timestamp, and the Merkle root node. The block body includes the current transaction record and all previous transaction records that have been successfully verified. It is the basis for data not to be tampered with and traceable.

The main participants in the fresh food e-commerce supply chain include producers (individual farmers, fresh food bases, etc.), processing companies, wholesalers, logistics companies, fresh food e-commerce platform companies and consumers, etc. At present, there are many fresh electricity business enterprises, including $\mathrm{O} 2 \mathrm{O}$ mode of Hema fresh, B2C of Yiguo fresh, and epidemic induced community group Meituan preferred and other electricity business models. For example, in the supply chain link of selected fresh food e-commerce for tadpoles, suppliers (fresh food bases, manufacturers and distributors, etc.) transport goods to e-commerce enterprise warehouses, and e-commerce enterprises complete logistics distribution. For most fresh food e-commerce supply chains, see Figure 3.

At present, fresh food e-commerce companies have weak control over the upstream of the supply chain. Most of the suppliers are farmers and agricultural cooperatives, whose level of informatization is not high, and it is difficult to guarantee production standardization and product quality. During the circulation of fresh products, packaging and logistics costs are relatively high. Most of the manufacturers and wholesalers undertake logistics and transportation by themselves. The logistics enterprises are also chaotic, showing many, small, scattered characteristics. It is not possible to form a situation of cooperative development, which restricts the efficiency of cold chain operations. In the fresh food e-commerce supply chain, the rights and information of each subject are asymmetrical, and there is a lack of trust mechanisms and information sharing. The quality of the partnership is not high, lacking integration and coordination capabilities. The actual customer experience is poor, and the price of fresh products is not easy to accept by customers. The value concept needs to be transformed. The consortium blockchain is quite in line with the development direction of coordination, integration, and standardization of fresh food e-commerce supply chain. Therefore, we build a fresh food e-commerce blockchain information platform based on the consortium blockchain to achieve controllable product quality, traceability of logistics, trust building, information sharing and reasonable distribution of benefits, etc., so as to improve the performance of the fresh food e-commerce supply chain (see Figure 4). 


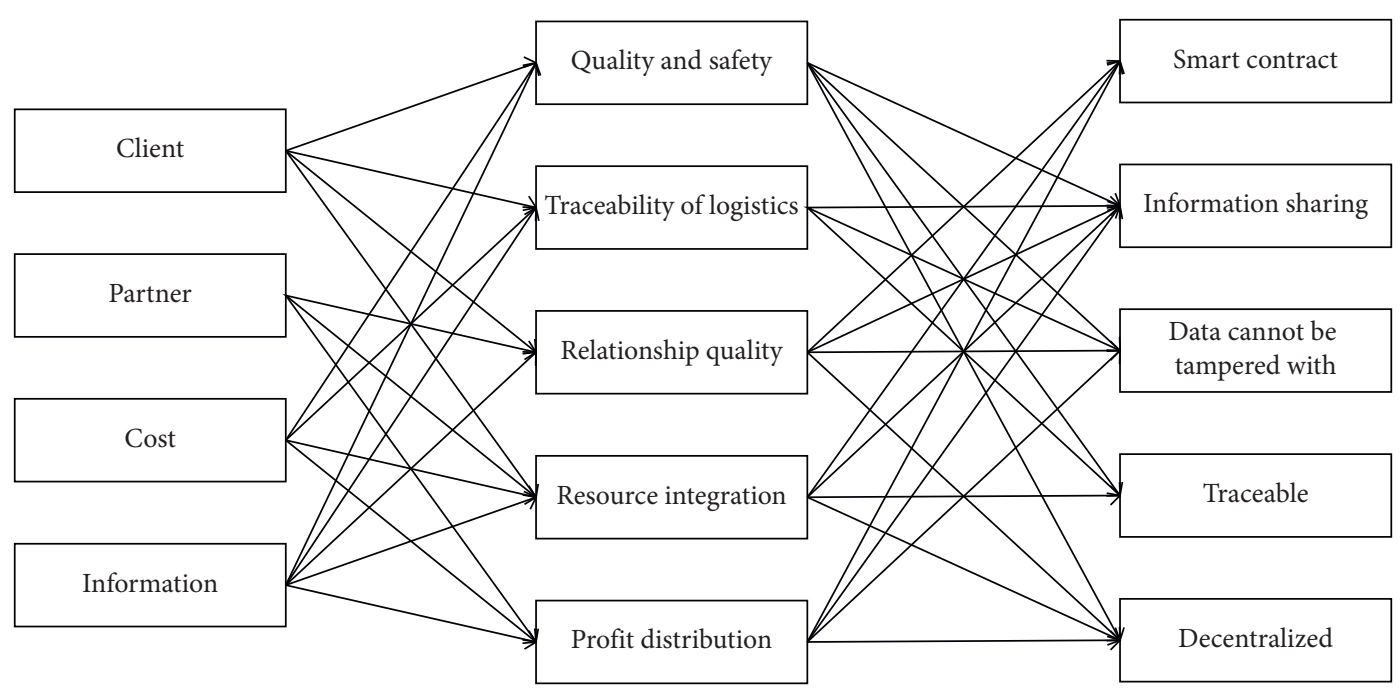

Internal and external
factors $\longrightarrow$ Raise demand $\longrightarrow \begin{gathered}\text { Blockchain technology } \\ \text { characteristics }\end{gathered}$

FIGURE 1: Blockchain technology characteristics and fresh food e-commerce supply chain performance optimization demand coupling.

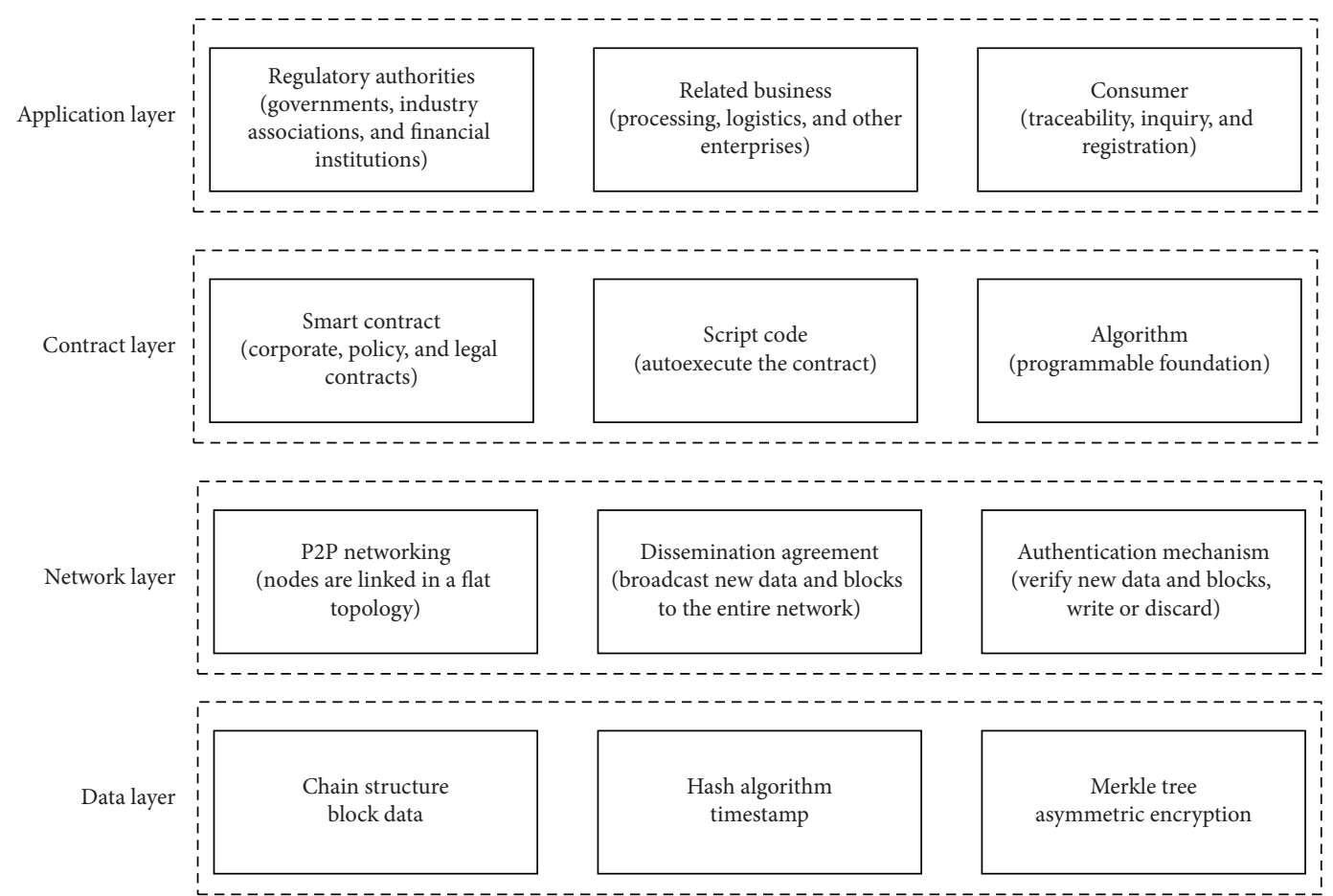

FIGURE 2: Infrastructure of blockchain information platform.

$\stackrel{\text { Logistics }}{\longrightarrow}$

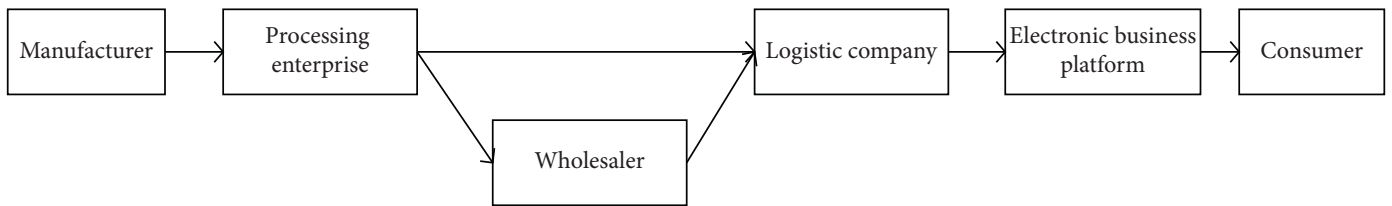

Figure 3: Fresh food e-commerce supply chain. 


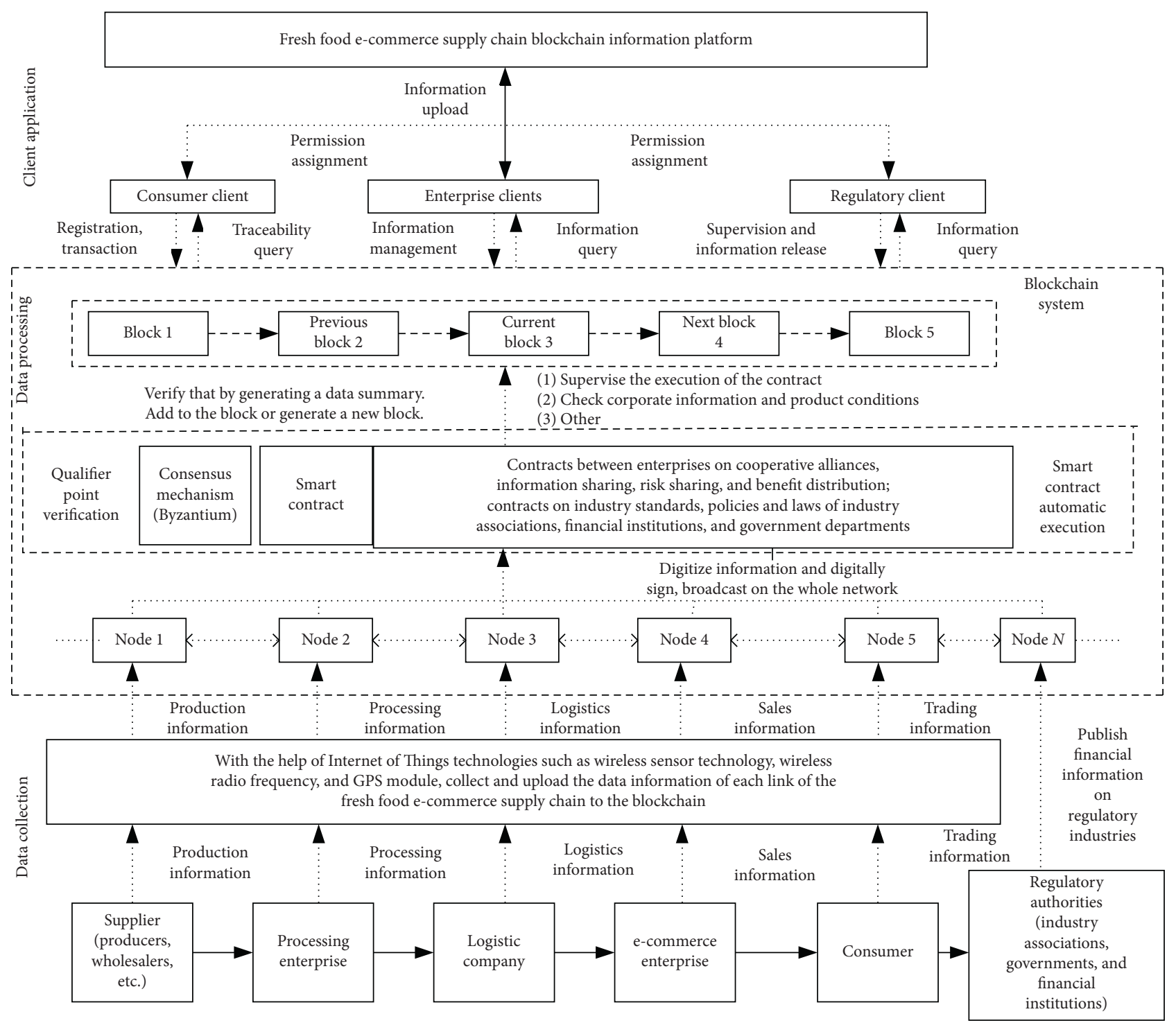

FIGURE 4: Blockchain information platform of fresh food e-commerce supply chain based on alliance chain.

The construction of the blockchain information platform for the fresh food e-commerce supply chain must unite the various components of the supply chain (suppliers, processing companies, logistics companies, e-commerce companies, etc.), fresh food industry associations, government regulatory agencies, and financial institutions, and the core enterprises of fresh food e-commerce should take the lead to build a consortium blockchain with multiple types of companies and departments as nodes. The blockchain information platform is divided into three parts: data collection, data processing, and client applications.

In the data collection stage, various enterprise systems and contracts are formulated in accordance with unified standards. The composition subject of the fresh food e-commerce supply chain collects and uploads production information (product variety, growth environment, drug utilization and growth time, etc.), processing information (processing unit, processing flow, quarantine results, etc.), logistics information (carrier, vehicle information, delivery route, cabin temperature and estimated time, etc.), warehousing information (product quantity, storage time, storage humidity, temperature and product freshness, etc.), sales information (sales time, sales price, sales quantity, product warranty period, etc.), and transaction information (consumer order time, payment information and purchase category, etc.) through technologies such as Internet of Things technology (wireless sensors, radio frequency, and GPS), video surveillance, and QR codes, and each subject is distributed and linked in the blockchain through P2P networking. After the information is uploaded, it will be broadcast on the whole network. After it is verified, the data is stored in each node in a distributed manner, and RFID tags or QR codes can be generated at each stage to facilitate data collection and query. The decentralized and distributed storage method has transformed the traditional trust mechanism from trusting people to trusting machines.

In the data processing stage, companies upload information about their internal capabilities, fresh products, and 
transactions through the nodes they control, which will be hashed to generate a digital summary and digitize the information. The node digitally signs the digital summary and broadcasts it to the entire network. Other nodes generate a summary by hash algorithm and verify the authenticity of information by comparing the decrypted digital summary. Through the Byzantine consensus algorithm, the three-phase consensus is pre-prepared, prepared, and submitted. After the verification node is time stamped, the successfully verified data is input into the blockchain. After the data is successfully verified, the smart contract of the system will automatically execute all verified agreements (including transaction price, benefit distribution, risk sharing, and other agreements). Asymmetric encryption, hash algorithm, and consensus mechanism ensure that the information can not be tampered with and is safe and reliable. The chain structure of the block, timestamp, and Merkle tree make it possible to trace the data from the source to the end.

The applications at the client end serve consumers, enterprises and regulatory authorities, etc. Each participant obtains a public key and a private key through registration and is assigned different access. Consumers can trace the source of product information through the client end, monitor the operation of the blockchain network, and perform credit ratings on sellers, etc. Enterprises can manage and release corporate information, realize information sharing, coordinate and integrate corporate operations, and integrate resources to form a rapidly reorganized and reactive supply chain. The use of smart contracts can reduce transaction costs between enterprises and promote the establishment of long-term contractual relationships between enterprises. The regulatory authorities supervise and spotcheck whether fresh products meet standards through the client end and supervise corporate credit and the signing of smart contracts. When necessary, they can enforce the implementation of smart contracts such as benefit sharing and risk sharing.

The consortium blockchain has technical features such as information traceability, smart contracts, and information sharing, which can promote the horizontal integration and vertical coordination of fresh food e-commerce, so that it can develop in the direction of integration and improve overall performance. First of all, the founding members of the consortium blockchain (core companies, various departments and institutions, etc.) work together to formulate the strategic goals of the fresh food e-commerce supply chain and generate smart contracts, which is added to the blockchain. That is, to formulate unified industry standards, enterprise operation systems (benefit distribution system and reward and punishment system) and collaboration integrate the degree and scope to meet the individual needs of customers, enhance consumer experience, and maximize customer value. Secondly, with the help of blockchain decentralization, distributed storage, and consensus mechanism, we can build trust among the main bodies of the fresh food e-commerce supply chain and promote the horizontal integration of all links in the fresh food e-commerce supply chain. In this way, it can not only reduce disorderly competition, integrate and share various resources and information, and form a large-scale operation, but also obtain large-scale economic effects and improve competitive position. Through the trust mechanism and information sharing provided by the blockchain, the main enterprises of the fresh food supply chain can coordinate vertically. Companies with different complementary capabilities can join forces and sign contracts to gain advantages in a competitive environment, thereby achieving common vision and goals. Finally, according to the formulated unified industry standards and system specifications, we can use the properties of blockchain technology such as nontampering, traceability, information sharing, smart contracts, and consensus mechanisms to build a blockchain information platform, ensure the safety and control of product quality in the fresh food e-commerce supply chain, achieve traceability of logistics information, promote the improvement of the quality of relationships between enterprises, and achieve resource integration and benefit distribution. As a result, the overall performance of fresh e-commerce supply chain can be improved.

\section{Supply Chain Performance Analysis before and after Applying Blockchain}

In order to study the changes of supply chain profits before and after the application of blockchain information platform (BIP) in the fresh e-commerce supply chain, we have simplified the fresh e-commerce supply chain into a secondary supply chain which was composed of fresh suppliers and e-commerce platforms in this section. In addition, suppliers could receive first-hand information such as freshness and production time in this two-level supply chain system, so they would like to obtain more contracts when misreporting product information. This study has set the research objects with the manufacturer as the leader and the e-commerce platform as the follower in decentralized supply chain. In the Stackelberg game model, the supplier releases the raw product with quality, safety, and reliability of $\beta$ when not using BIP and then sends the wholesale price $\omega$ and false production time $\gamma t$ to the e-commerce platform, so that the e-commerce platform could determine the order quantity $Q$ based on the wholesale price $\omega$ along with the retail price $P$.

4.1. Variable Description. When fresh e-commerce secondary supply chain has acted as a profit system, the pursuit of profit maximization, consumer order quantity will be affected by price, freshness, and safety. As shown in Figure 5, variables in the corresponding basic model are assumed as follows: $P$ is the unit retail price of the e-commerce platform; $\omega$ is the unit wholesale price of raw products provided by the supplier; $c_{s}$ is the unit production cost and transportation cost of the supplier; $c_{r}$ is the unit storage and distribution cost of the e-commerce platform; $t$ is the production time of real fresh products ( $\gamma$ is a misreporting factor, and $\gamma \in[0,1]$; then the misreporting of production time is $\gamma t) ; \theta_{(t)}$ and $\theta_{(\gamma t)}$ are the freshness of real fresh products and product freshness under false reporting behavior, respectively (according to Chenet al. [26], freshness of raw products $\theta_{(t)}=1-t^{2} / \mathrm{T}^{2}$, $0 \leq t \leq \mathrm{T}, \mathrm{T}$ is the life cycle); $\beta$ is the quality reliability of fresh 


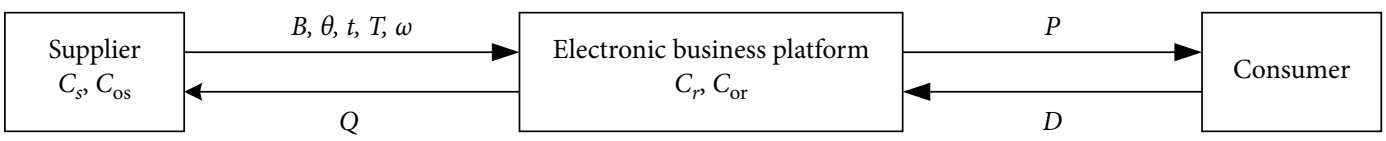

Figure 5: Fresh food e-commerce supply decision flow chart.

products; $c_{\mathrm{os}}$ and $c_{\mathrm{or}}$ are the costs of suppliers and e-commerce platforms after using BIP, respectively; $Q$ and $Q^{\circ}$ are the order quantities of retailers under false reporting and true freshness, respectively; $D$ and $D^{o}$ are market demand forecast under false reporting behavior and true freshness, respectively; the e-commerce platform would place orders to suppliers according to the demand forecast, so $Q=D, D^{o}=Q^{o}$.

The supplier lied about freshness and the reliability of product quality was low when not using BIP. The reliability coefficient was represented by $\lambda(0 \leq \lambda \leq 1)$. While blockchain technology will reduce misreporting after using BIP, therefore improving trust relationship and increasing consumer purchasing power, then reliability coefficient would reach $\lambda^{B}>\lambda$ and $P^{B}$ is the unit retail price of the e-commerce platform after using BIP; $\omega^{B}$ is the unit wholesale price of fresh products provided by the supplier after using BIP; $Q^{B}=Q^{o}$ is the order quantity of e-commerce platform after using BIP; $\pi_{r}$ and $\pi_{s}$ are the profits of suppliers and e-commerce platforms when not using BIP; $\pi_{s}^{B}$ and $\pi_{r}^{B}$ are the profits of suppliers and e-commerce platforms after using BIP.

\subsection{Model Assumptions}

Assumption 1. The supplier provides a fresh product for the e-commerce platform with sufficient production capacity. Both parties aim at maximizing their own interests which are risk-neutral and completely rational.

Assumption 2. Supply chain members need to improve food quality and safety partnership through blockchain information platform; consumers are willing to pay the right amount for safe, reliable, and fresh products.

Assumption 3. Fresh product order demand is affected by price, freshness, and reliability. According to the literature of Zeng et al. [27] and Pan Liu [28], the demand function is assumed to be as $D=a-b p+\lambda \beta \theta_{(t)} ; a$ is the market size, and $b$ is the price sensitivity coefficient ( $a$ and $b$ are constants greater than 0 ; we set $a$ and $b$ as 1 , for better calculation so that the simplified demand function is $\left.Q=1-p+\lambda \beta \theta_{(t)}\right)$.

\subsection{Model Building and Solving}

4.3.1. Set Up Decision Model When Not Using the Blockchain Information Platform (BIP). The supplier conceals the raw product information to the e-commerce platform when not using BIP, prompting the e-commerce platform to increase the order quantity. At this point, the demand function is

$$
Q=1-p+\lambda \beta \theta_{(\gamma t)}
$$

At this point, the profit function of suppliers and fresh e-commerce platform are

$$
\begin{aligned}
& \pi_{s}=\left(\omega-c_{s}\right) \cdot \mathrm{Q}, \\
& \pi_{r}=\left(P-\omega-c_{r}\right) \cdot Q^{o}-\left(Q-Q^{O}\right) \cdot \omega .
\end{aligned}
$$

Based on equations (1) and (3), we have solved this problem by inverse induction. Firstly, the second derivative of $Q$ in (3) $\alpha^{2} \pi_{r} / \alpha^{2} Q=-2<0$, so there is an optimal solution. Set the first derivative to be $\alpha \pi_{r} / \alpha Q=0$, and the optimal values are

$$
\begin{aligned}
& Q^{*}=\frac{1+2 \lambda \beta \theta_{(\gamma t)}-\lambda \beta \theta_{(t)}-\omega-c_{r}}{2}, \\
& \mathrm{P}^{*}=\frac{1+\lambda \beta \theta_{(t)}+c_{r}+\omega}{2} .
\end{aligned}
$$

Substitute equations (4) and (5) into equation (2). The second derivative of $\omega$ is $\alpha^{2} \pi_{s} / \alpha^{2} \omega=-1<0$, so there is an optimal solution. Set the first derivative to be $\alpha \pi_{s} / \alpha \omega=0$, and the optimal value of $\omega$ is obtained as

$$
\omega^{*}=\frac{1+2 \lambda \beta \theta_{(\gamma t)}-\lambda \beta \theta_{(t)}-c_{r}+c_{s}}{2} .
$$

Substituting equation (6) into equations (4) and (5), the optimal order quantity and retail price can be obtained as follows:

$$
\begin{aligned}
Q^{*} & =\frac{1+2 \lambda \beta \theta_{(\gamma t)}-\lambda \beta \theta_{(t)}-c_{s}-c_{r}}{4}, \\
P^{*} & =\frac{3+2 \lambda \beta \theta_{(\gamma t)}+\lambda \beta \theta_{(t)}+c_{r}+c_{s}}{4} .
\end{aligned}
$$

Finally, put $Q^{*}, P^{*}$, and $\omega^{*}$ into equations (1) and (2), respectively, to solve the optimal profit of the supplier and the e-commerce platform; then the profit of the whole chain is, respectively: 


$$
\begin{aligned}
& \pi_{s}^{*}=\frac{\left[1+2 \lambda \beta \theta_{(\gamma t)}-\lambda \beta \theta_{(t)}-c_{s}-c_{r}\right]^{2}}{8}, \\
& \pi_{r}^{*}=\frac{\left[1-2 \lambda \beta \theta_{(\gamma t)}+3 \lambda \beta \theta_{(t)}-c_{r}-c_{s}\right]^{2}}{16}+\frac{\left(\lambda \beta \theta_{(t)}-\lambda \beta \theta_{(\gamma t)}\right) \cdot\left(1+2 \lambda \beta \theta_{(\gamma t)}-\lambda \beta \theta_{(t)}-c_{r}+c_{s}\right)}{2} .
\end{aligned}
$$

Total profit:

$$
\begin{aligned}
\pi=\pi_{s}^{*}+\pi_{r}^{*}= & \frac{\left[1+2 \lambda \beta \theta_{(\gamma t)}-\lambda \beta \theta_{(t)}-c_{s}-c_{r}\right]^{2}}{8}+\frac{\left[1-2 \lambda \beta \theta_{(\gamma t)}+3 \lambda \beta \theta_{t}-c_{r}-c_{s}\right]^{2}}{16} \\
& +\frac{\left(\lambda \beta \theta_{(t)}-\lambda \beta \theta_{(\gamma t)}\right) \cdot\left(1+2 \lambda \beta \theta_{(\gamma t)}-\lambda \beta \theta_{(t)}-c_{r}+c_{s}\right)}{2} .
\end{aligned}
$$

Proposition 1. he improvement of freshness $\theta_{(\gamma t)}$, the profit of suppliers $\pi_{s}^{*}$ will increase when not using BIP, the profit of e-commerce platform $\pi_{r}^{*}$ will decrease, and the overall profit of fresh e-commerce supply chain $\pi$ will decrease.

Proof. Calculate $\theta_{(\gamma t)}$ for equations (9) and (10) to obtain the first derivative. Combined with equations (6)-(8), it can be seen that

$$
\begin{aligned}
Q^{o}= & 1-2 \lambda \beta \theta_{(\gamma t)}+3 \lambda \beta \theta_{(t)}-c_{s}-c_{r}>0 ; \theta_{(\gamma t)}>\theta_{(t)}, \\
& 1+2 \lambda \beta \theta_{(\gamma t)}-\lambda \beta \theta_{(t)}-c_{s}-c_{r}>0 ; 1+2 \lambda \beta \theta_{(\gamma t)} \\
& -\lambda \beta \theta_{(t)}-c_{s}-c_{r}>0 .
\end{aligned}
$$

Then, $\alpha \pi_{s}^{*} / \alpha \theta_{(\gamma t)}=\lambda \beta / 2 \cdot\left(1+2 \lambda \beta \theta_{(\gamma t)}-\lambda \beta \theta_{(t)}-c_{s}-c_{r}\right)>0$;

$$
\begin{aligned}
\frac{\alpha \pi_{r}^{*}}{\alpha \theta_{(\gamma t)}}= & \frac{-\lambda \beta}{4} \cdot\left(1-2 \lambda \beta \theta_{(\gamma t)}+3 \lambda \beta \theta_{(t)}-c_{s}-c_{r}\right) \\
& +2 \lambda \beta \cdot\left(\lambda \beta_{(t)}-\lambda \beta_{(\gamma t)}\right)-\lambda \beta \cdot\left(1+2 \lambda \beta \theta_{(\gamma t)}-\lambda \beta \theta_{(t)}-c_{r}+c_{s}\right)<0, \\
\frac{\alpha \pi}{\alpha \theta_{(\gamma t)}}= & \frac{-\lambda \beta}{4} \cdot\left(1-2 \lambda \beta \theta_{(\gamma t)}+3 \lambda \beta \theta_{(t)}-c_{s}-c_{r}\right) \\
& +2 \lambda \beta \cdot\left(\lambda \beta_{(t)}-\lambda \beta_{(\gamma t)}\right)-\frac{\lambda \beta}{2} \cdot\left(1+2 \lambda \beta \theta_{(\gamma t)}-\lambda \beta \theta_{(t)}-c_{r}+c_{s}\right)<0 .
\end{aligned}
$$

The value of function $\pi_{s}^{*}$ would increase with the expanding of $\theta_{(\gamma t)}$, and the value of $\pi_{r}^{*}$ and the value of function $\pi$ would decreases. Then Proposition 1 will be proved.

4.3.2. Set Up Decision Model When Applying Blockchain Information Platform (BIP). Misreporting behavior will be resolved when using BIP, and the cost consumed by the supplier and the e-commerce platform is reduced to $c_{s}^{B}$ and $c_{r}^{B}\left(c_{s}>c_{s}^{B}, c_{r}>c_{r}^{B}\right)$. At this point, the demand function of the e-commerce platform is

$$
Q^{B}=1-P+\lambda \beta \theta_{(t)}
$$

Profit functions of suppliers and e-commerce platforms are

$$
\begin{aligned}
& \pi_{s}^{B}=\left(\omega^{B}-c_{s}^{B}-c_{\mathrm{os}}\right) \cdot \mathrm{Q}^{B}, \\
& \pi_{r}^{B}=\left(\mathrm{P}^{B}-c_{r}^{B}-c_{\mathrm{or}}-\omega^{B}\right) \cdot \mathrm{Q}^{B} .
\end{aligned}
$$

We also take the first derivative and the second derivative with respect to $Q^{B}$ by backward induction. Firstly, $\alpha^{2} \pi_{r}^{B} / \alpha^{2} Q^{B}=-2<0$, so there is an optimal solution. Set the first derivative to be $\alpha \pi_{r}^{B} / \alpha Q^{B}=0$, thus acquiring $Q^{B *}$ and $\mathrm{P}^{B *}$. Substitute $\mathrm{Q}^{B *}$ into equation (15), and calculate the first and second derivatives of $\omega^{B}, \alpha^{2} \pi_{s}^{B} / \alpha^{2} \omega^{B}=-1<0$, so there is an optimal solution. Set $\alpha \pi_{s}^{B} / \alpha \omega^{B}=0$, and the optimal wholesale price when using BIP is obtained as

$$
\omega^{B *}=\frac{1+\lambda^{B} \beta \theta_{(t)}-c_{r}^{B}-c_{\mathrm{or}}+c_{s}^{B}+c_{\mathrm{os}}}{2} .
$$

Substitute $\omega^{B}$ into $Q^{B *}$ and $\mathrm{P}^{B *}$ to get the optimal order quantity and retail price as

$$
\begin{aligned}
\mathrm{Q}^{B *} & =\frac{1+\lambda^{B} \beta \theta_{(t)}-c_{r}^{B}-c_{\mathrm{or}}-c_{s}^{B}-c_{\mathrm{os}}}{4}, \\
\mathrm{P}^{B *} & =\frac{3+3 \lambda^{B} \beta \theta_{(t)}+c_{r}^{B}+c_{\mathrm{or}}+c_{s}^{B}+c_{\mathrm{os}}}{4} .
\end{aligned}
$$

Substituting equations (17)-(19) into equations (15) and (16), it can be obtained that the profit of the supplier and the profit of the e-commerce platform after using the blockchain information platform are (20) and (21), respectively:

$$
\begin{aligned}
& \pi_{s}^{B *}=\frac{\left(1+\lambda^{B} \beta \theta_{(t)}-c_{r}^{B}-c_{\mathrm{or}}-c_{s}^{B}-c_{\mathrm{os}}\right)^{2}}{8}, \\
& \pi_{r}^{B *}=\frac{\left(1+\lambda^{B} \beta \theta_{(t)}-c_{r}^{B}-c_{\mathrm{or}}-c_{s}^{B}-c_{\mathrm{os}}\right)^{2}}{16} .
\end{aligned}
$$

The overall profit of fresh electricity supply chain is 


$$
\pi^{B *}=\frac{3\left(1+\lambda^{B} \beta \theta_{(t)}-c_{r}^{B}-c_{\mathrm{or}}-c_{s}^{B}-c_{\mathrm{os}}\right)^{2}}{16}
$$

Proposition 2. With the improvement of product reliability coefficient $\lambda^{B}$ and freshness $\theta_{(t)}$, the profits of all parties in the supply chain of fresh e-commerce suppliers and the overall profits would increase.

Proof. Find the first-order partial derivatives of $\lambda^{B}$ and $\theta_{(t)}$ with respect to equations (20)-(22), and then combine equation (18); we can obtain

$$
\begin{aligned}
& \frac{\alpha^{2} \pi_{s}^{B *}}{\alpha^{2} \lambda^{B}}=\frac{\beta \theta_{(t)}}{4} \cdot\left(1+\lambda^{B} \beta \theta_{(t)}-c_{r}^{B}-c_{\mathrm{or}}-c_{s}^{B}-c_{\mathrm{os}}\right)>0, \\
& \frac{\alpha^{2} \pi_{s}^{B *}}{\alpha^{2} \theta_{(t)}}=\frac{\lambda \beta}{4} \cdot\left(1+\lambda^{B} \beta \theta_{(t)}-c_{r}^{B}-c_{\mathrm{or}}-c_{s}^{B}-c_{\mathrm{os}}\right)>0 .
\end{aligned}
$$

After observing, $\alpha^{2} \pi_{r}^{B *} / \alpha^{2} \lambda^{B}=$ 式(21)/2>0, $\alpha^{2} \pi_{r}^{B *} / \alpha^{2}$ $\theta_{(t)}=$ 式 $(22) / 2>0 ; \alpha^{2} \pi^{B *} / \alpha^{2} \lambda^{B}=$ 式 $(21) \times 3 / 2>0, \alpha^{2} \pi^{B *} /$ $\alpha^{2} \theta_{(t)}=$ 式 $(21) \times 3 / 2>0$ 。 Therefore, as the values of $\lambda^{B}$ and $\theta_{(t)}$ increase, the values of functions $\pi_{s}^{B *}, \pi_{r}^{B *}$ 和 $\pi^{B *}$ would expand. Then Proposition 2 will be proved.

Proposition 3. When $c_{o r}+c_{o s}<\min \left(\mu_{1}, \mu_{2}\right)$, the whole fresh e-commerce supply chain and the profits of all parties increase with the application of the blockchain information platform.

Proof. $\pi_{s}^{B}>\pi_{s} \pi_{r}^{B}>\pi_{r}$, the profits of suppliers and retailers after using BIP are greater than the profits before using BIP.

$$
\begin{aligned}
\pi_{s}^{B}-\pi_{s}= & \frac{\left(1+\lambda^{B} \beta \theta_{(t)}-c_{r}^{B}-c_{\mathrm{or}}-c_{s}^{B}-c_{\mathrm{os}}\right)^{2}}{8} \\
& -\frac{\left[1+2 \lambda \beta \theta_{(\gamma t)}-\lambda \beta \theta_{(t)}-c_{s}-c_{r}\right]^{2}}{8},
\end{aligned}
$$

当 $c_{\mathrm{or}}+c_{\mathrm{os}}<\left(\lambda+\lambda^{B}\right) \beta \theta_{(t)}-2 \lambda \beta \theta_{(\lambda t)}+c_{s}-c_{s}^{B}+c_{r}-c_{r}^{B}$ $=\mu_{1}, \pi_{s}^{B}-\pi_{s}>0$

$$
\pi_{r}^{B}-\pi_{r}=\frac{\left(1+\lambda^{B} \beta \theta_{(t)}-c_{r}^{B}-c_{\mathrm{or}}-c_{s}^{B}-c_{\mathrm{os}}\right)^{2}}{16}-\frac{\left[1-2 \lambda \beta \theta_{(\gamma t)}+3 \lambda \beta \theta_{(t)}-c_{r}-c_{s}\right]^{2}}{16}-\frac{\left(\lambda \beta \theta_{(t)}-\lambda \beta \theta_{(\gamma t)}\right) \cdot\left(1+2 \lambda \beta \theta_{(\gamma t)}-\lambda \beta \theta_{(t)}-c_{r}+c_{s}\right)}{2} .
$$

We have

$$
c_{o r}+c_{o s}<\lambda^{B} \beta \theta_{(t)}+1-c_{r}^{B}-c_{s}^{B}-\sqrt{\left[1-2 \lambda \beta \theta_{(\gamma t)}+3 \lambda \beta \theta_{t}-c_{r}-c_{s}\right]^{2}+8\left(\lambda \beta \theta_{(t)}-\lambda \beta \theta_{(\gamma t)}\right) \cdot\left(1+2 \lambda \beta \theta_{(\gamma t)}-\lambda \beta \theta_{(t)}-c_{r}+c_{s}\right)}=\mu_{2}, \quad \pi_{r}^{B}-\pi_{r}>0 .
$$

Therefore, when $c_{\mathrm{or}}+c_{\mathrm{os}}<\min \left(\mu_{1}, \mu_{2}\right)$, all parties and overall profits of fresh e-commerce supply chain blockchain information platform will be improved.

Proposition 4. With the increase of the product reliability coefficient $\lambda$, before and after the application of BIP, the wholesale prices of suppliers and the retail prices of retailers in the fresh food e-commerce supply chain both increase.

Proof. Finding the first-order partial derivative of $\lambda$ with respect to equations (6), (8), (17), and (19), we can obtain

$$
\begin{aligned}
& \frac{\partial \omega^{*}}{\partial \lambda}=\beta \theta_{(\gamma t)}-\frac{\beta \theta_{t}}{2}>0, \\
& \frac{\partial \mathrm{P}^{*}}{\partial \lambda}=\frac{\beta \theta_{(\gamma t)}}{2}-\frac{\beta \theta_{t}}{4}>0, \\
& \frac{\partial \omega^{B *}}{\partial \lambda^{B}}=\frac{\beta \theta_{t}}{2}>0, \\
& \frac{\partial \mathrm{P}^{B *}}{\partial \lambda^{B}}=\frac{3 \beta \theta_{t}}{4}>0 .
\end{aligned}
$$

Therefore, as the freshness $\lambda$ of fresh products increases, the retail prices of fresh food supply chain suppliers will increase before and after the application of BIP, and the retail prices of fresh food e-commerce platforms will also increase. It proves that people are willing to pay higher prices for products with a high level of greenness and product quality.

It is found that the behavior of suppliers hiding product freshness information will damage the profit of the e-commerce platform and the overall performance of the supply chain through game analysis on the changes of each member and overall profit of fresh e-commerce supply chain before and after application of blockchain information platform. When using the blockchain information platform, the application cost is within a certain range, which can enhance the trust among members, increase the reliability of product information, and inhibit the behavior of misreporting. It can not only improve the profit of suppliers and e-commerce platform, but also promote the overall performance with the overall profit of the fresh supply chain.

When using the blockchain information platform, the application cost is within a certain range, which can improve the trust among members, increase the reliability of product information, and suppress false reporting behavior. It can improve not only the profits of 
suppliers and e-commerce platforms, but also the overall profits of the fresh supply chain. It will also increase and improve the overall performance of the fresh e-commerce supply chain. According to $\mathrm{Fu}$, Yonggui, and Jianming Zhu (2021), blockchain technology will provide new ideas for the fresh food e-commerce supply chain [29]. According to Karamachoski (2020) blockchain technology will bring a disruption in plenty of industries and businesses [30].

\section{Numerical Analysis}

Assuming that a manufacturer and an e-commerce platform form a secondary fresh e-commerce supply chain, they will all adopt BIP and the market demand will be greater than zero, while the actual situation will be modified after referring to the literature data of Zeng Youxin [33] and Pan Liu[34]. If setting $\beta=3, \theta_{(t)}=0.2, \theta_{(\gamma t)}=0.25, \quad c_{s}=0.13, \quad c_{r}=0.15, \quad c_{\mathrm{os}}=0.01$, $c_{\mathrm{or}}=0.02, c_{s}^{B}=0.01, c_{r}^{B}=0.03$, combining with (9), (10), (20), and (21), we can see from Figure 6 that, in the two models without using BIP and after using BIP, suppliers and (retailers) fresh e-commerce platform revenue would increase with the improvement of the reliability coefficient $\lambda$ of raw product quality information. Their benefits after using BIP are greater than those without using blockchain information platform (the investment cost of blockchain is within the range of Proposition 3). Besides, the great trust relationship between consumers, e-commerce platforms, and suppliers will be improved when using blockchain information platform. Consumers are more assured about the products and platform, and the platform is more convenient for the management and cooperation. Moreover, their overall profits would increase with the improvement of product reliability coefficient and freshness, thus enhancing supply chain efficiency. Blockchain technology has brought improvement to the performance of the whole supply chain.

The reliability of the product after using BIP is greater than that without using it based on the assumed information, so we assumed $\lambda^{B}=0.6$ and $\lambda=0.4$. At the same time, considering the function of the optimal demand in both the unused and applied BIP models, we obtain Figure 7, which shows the trend of diversification as the cost of investing in BIP would increase; see the chart below. As the BIP cost paid by retailers and suppliers increases, the order quantity and the revenue of retailers will decrease. These results suggest that if retailers and manufacturers want to gain more revenue after using BIP, they should minimize the cost of investment in BIP and reduce the cost of technology as much as possible. Blockchain landing applications need to make progress immediately to reduce costs and make the front and back ends of the supply chain acceptable.

As shown in Figure 8, suppliers and e-commerce platforms will continuously increase their wholesale prices and retail prices, respectively, with the increase in BIP investment costs of fresh suppliers and e-commerce platforms. Both wholesale price and retail price would change from low to high before and after using BIP. Therefore, the cost of BIP investment needs to be shared by

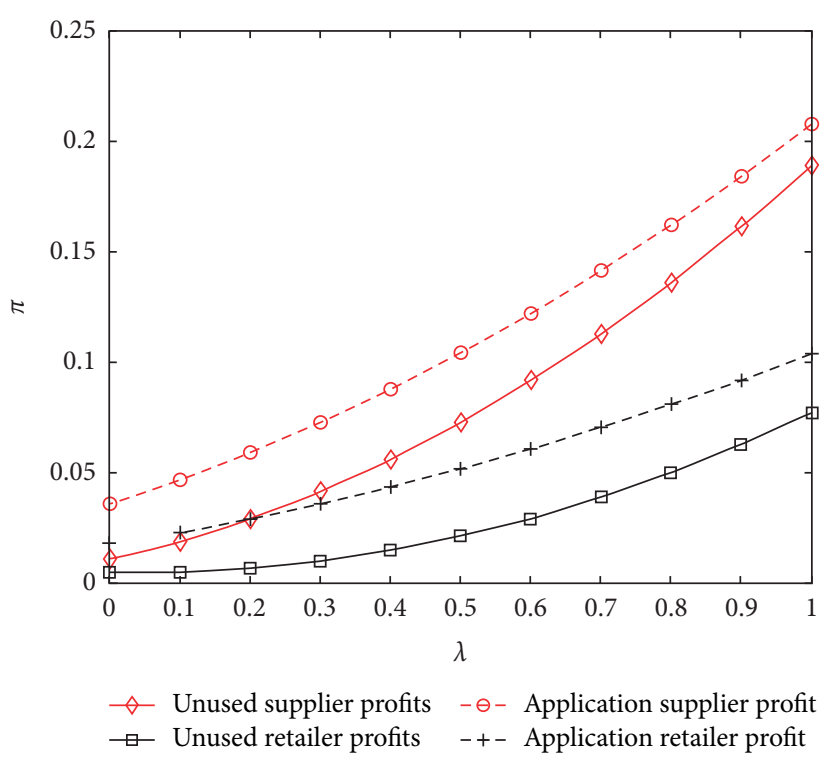

FIgURE 6: Relationship between supplier and retailer profit and reliability.

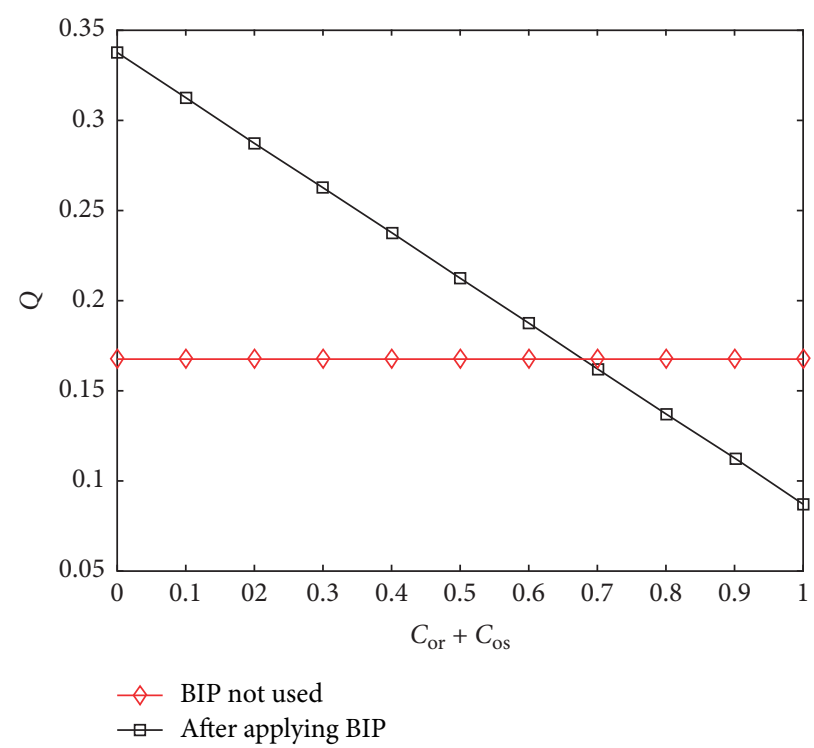

FIgURE 7: Relationship between fresh food e-commerce order quantity and investment BIP cost.

all members. The decrease of order quantity with the increase of investment cost is also directly related to the change of price. Therefore, since fresh e-commerce supplier is the core enterprise in the supply chain, it should actively coordinate the information between the members, promote the implementation of information sharing + cost sharing contract, make the investment BIP cost-effectively reduced, so as to increase the supply chain surplus and conduct appropriate pricing decisions.

\section{Conclusion}

The development of the fresh food e-commerce supply chain is gradually moving towards a healthy, green, integrated, and 


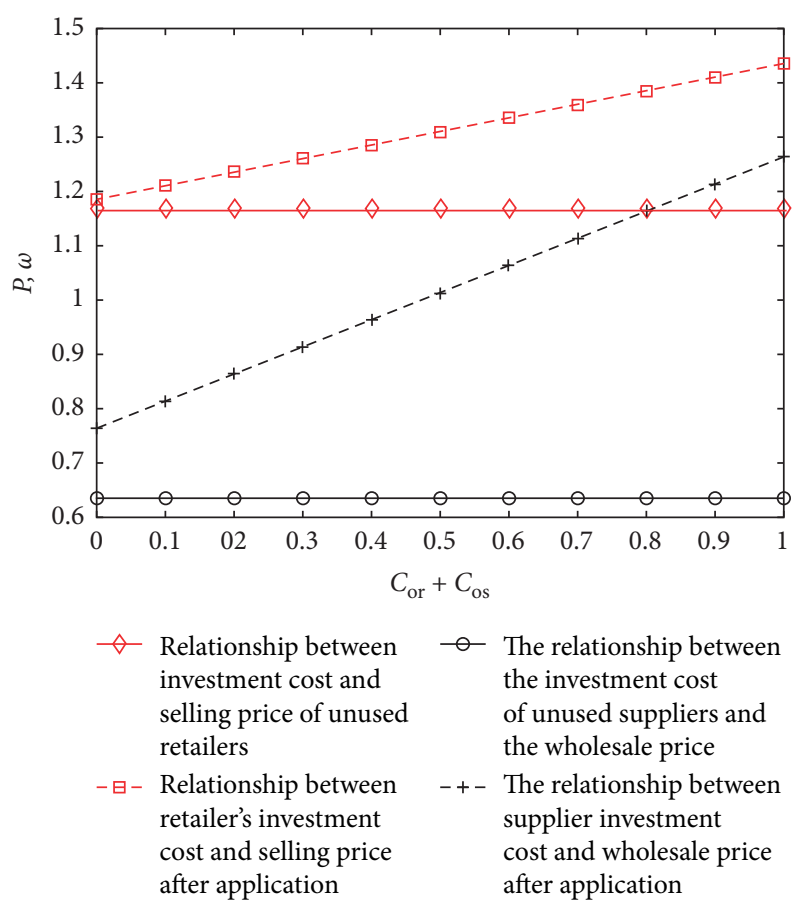

FIGURE 8: Retailer (e-commerce platform) and supplier investment BIP cost and price relationship diagram.

sustainable stage. The core competitiveness of price discounts and distribution services is not enough to support the long-term development of the fresh food e-commerce supply chain. With the development and maturity of informatization and digital technology, the digitization and informatization of the fresh food e-commerce supply chain is an important direction for fresh food e-commerce to improve quality and efficiency. The application research of blockchain technology in various fields is gradually strengthened. With its features such as tamper-resistant data, traceability, information sharing, smart contracts, and consensus mechanisms, it can optimize internal and external factors such as food quality and safety issues, supply chain partnerships, supply chain collaboration and integration, actual customer experience, and information technology, ensure the safety and control of product quality in the fresh food e-commerce supply chain, achieve traceability of logistics information, promote the improvement of the quality of relationships between enterprises, and achieve resource integration and benefit distribution. As a result, the overall performance of fresh e-commerce supply chain can be improved.

This paper analyzes the coupling relationship between the characteristics of the blockchain technology and the factors that affect the performance improvement of fresh food e-commerce and designs a fresh food supply chain information platform based on the consortium blockchain to achieve the overall performance improvement of all parties in the chain. By constructing a Stackelberg game model between suppliers and e-commerce platforms, this article analyzes how blockchain technology affects the reliability of fresh product information and how it affects suppliers behavior of concealing the freshness of fresh products and studies and compares the profit changes of fresh food e-commerce supply chain before and after the application of blockchain information platform to verify the effect of blockchain technology on the performance improvement of fresh food e-commerce supply chain. According to the research results, when the cost of investing in a blockchain information platform is controlled within a certain range, it can not only improve the reliability of fresh products, but also restrain the behavior of concealment and improve the profit and overall performance of the main participants of the fresh food e-commerce supply chain.

\section{Data Availability}

The data used to support the findings of this study are included within the article(Numerical Analysis section).

\section{Conflicts of Interest}

The authors declare that there are no conflicts of interest.

\section{Acknowledgments}

This paper was financially supported by the 100 Outstanding Innovative Talents Support Project of Hebei Province (SLRC2019005), Social Science Foundation Project of Hebei Province (HB20GL011), and Soft Science Project of Hebei Science and Technology Department (20554702D) which made the present work possible.

\section{References}

[1] W. Liu, "Study on consumers," Food Safety Risk Perception and Purchasing Behavior under Fresh e-Commerce, Wuhan University of Technology, Wuhan, China, 2019.

[2] J. Shang and X. Ji, "Optimization of ecological agricultural supply chain based on block chain application," Journal of South China Agricultural University (Social Science Edition), vol. 19, no. 4, pp. 67-75, 2020.

[3] X. Zhou, "Influencing factors and optimization measures of supply chain overall performance," Journal of South-Central University for Nationalities (Humanities and Social Sciences), vol. S1, pp. 41-42, 2004.

[4] J. Wu, "Research on supply chain management of autonomous fresh food E-commerce based on blockchain," Journal of Dali University, vol. 5, no. 1, pp. 123-128, 2020.

[5] J. G. A. J. Van der Vorst, A. J. M. Beulens, and P. van Beek, "Modelling and simulating multi-echelon food systems," European Journal of Operational Research, vol. 122, no. 2, pp. 354-366, 2000.

[6] V. Joby George and M. Pillai, "A study of factors affecting supply chain performance," ICAME, vol. 2, pp. 018018012018, 2019.

[7] M. Zan, G. Chen, and Z. Wang, "Development of E-commerce of fresh agricultural products in China: progress," Difficulties and strategies on Economic Problems, vol. 12, pp. 68-74, 2020.

[8] M. Moazzam, P. Akhtar, E. Garnevska, and N. E. Marr, "Measuring agri-food supply chain performance and risk through a new analytical framework: a case study of New 
Zealand dairy," Production Planning \& Control, vol. 29, no. 15, pp. 1258-1274, 2018.

[9] W. Odongo, M. Dora, A. Molnár, D. Ongeng, and X. Gellynck, "Performance perceptions among food supply chain members," British Food Journal, vol. 118, no. 7, pp. 1783-1799, 2016.

[10] S. Yadav, D. Garg, and S. Luthra, "Development of IoT based data-driven agriculture supply chain performance measurement framework," Journal of Enterprise Information Management, vol. 34, no. 1, pp. 292-327, 2020.

[11] S. Yadav and D. Garg, "Implementing technology to improve stakeholder fairness perception," 18th International Symposium on Horticultural Economics and Management, vol. 1132, pp. 105-112, 2016.

[12] R. Kaipia, I. Dukovska-Popovska, and L. Loikkanen, "Creating sustainable fresh food supply chains through waste Reduction," International Journal of Physical Distribution \& Logistics Management, vol. 43, no. 3, pp. 262-276, 2013.

[13] T.-M. Yeh, F.-Y. Pai, and L.-C. Wu, "Relationship stability and supply chain performance for SMEs: from internal, supplier, and customer integration perspectives," Mathematics, vol. 8, no. 11, p. 1902, 2020.

[14] S. Saberi, M. Kouhizadeh, J. Sarkis, and L. Shen, "Blockchain technology and its relationships to sustainable supply chain management," International Journal of Production Research, vol. 57, no. 7, pp. 2117-2135, 2019.

[15] J. J. Sikorski, J. Haughton, and M. Kraft, "Blockchain technology in the chemical industry: machine to machine electricity market," Applied Energy, vol. 195, pp. 234-246, 2017.

[16] X. Zhou and F. Zheng, "A research on the construction of agricultural product quality and safety traceability system based on the blockchain technology," Journal of Fujian Provincial Committee Party School of CPC (Fujian Academy of Governance), vol. 1, no. 3, pp. 113-117, 2019.

[17] K. Salah, N. Nizamuddin, R. Jayaraman, and M. Omar, "Blockchain-based soybean traceability in agricultural supply chain,” IEEE Access, vol. 7, pp. 73295-73305, 2019.

[18] N. Alzahrani, "Blockchain-based intelligent contract for factoring business in supply chains," Annals of Operations Research, vol. 1, 2020.

[19] P. Li, "Research on the circulation safety of fresh agricultural products in my country from the perspective of blockchain," Journal of Commercial Economics, vol. 10, pp. 146-149, 2020.

[20] K. O. Park, "A study on sustainable usage intention of blockchain in the big data era: logistics and supply chain management companies," Sustainability, vol. 12, no. 24, p. 10670, 2020.

[21] B. Mei, "China's agricultural product cold chain logistics model and development countermeasures based on blockchain technology," Journal of Commercial Economics, vol. 5, pp. 97-100, 2020.

[22] D. Tang and X. Zhuang, "Decision-making of supply chain finance based on blockchain accounts receivables transfer platform," Systems Engineering, vol. 37, no. 6, pp. 58-66, 2019.

[23] J. Gauthier, "Blockchain-based intelligent contract for factoring business in supply chains," Annals Of Operations Research, vol. 1, no. 1, p. 1, 2020.

[24] M. L. Nandi, S. Nandi, H. Moya, and H. Kaynak, "Blockchain technology-enabled supply chain systems and supply chain performance: a resource-based view," Supply Chain Management: An International Journal, vol. 25, no. 6, pp. 841-862, 2020.

[25] K. S. Hald and A. Kinra, "How the blockchain enables and constrains supply chain performance," International Journal of Physical Distribution \& Logistics Management, vol. 49, no. 4, pp. 376-397, 2019.

[26] H. Chen, X. Jia, and M. Jiang, "Research on deision-making of fresh agricultural supply chain with blockchain under behavior of misreportin," Computer Engineering and Applications, vol. 55, no. 16, pp. 265-270, 2019.

[27] Y. Zeng, P. Yuan, and Y. Zhang, "Decision-making analysis of fresh E-commerce supply chain with different dominant powers from the perspective of game theory," Journal of Nanjing Audit University, vol. 16, no. 5, pp. 55-64, 2019.

[28] P. Liu, Y. Long, H.-C. Song, and Y.-D. He, "Investment decision and coordination of green agri-food supply chain considering information service based on blockchain and big data," Journal Of Cleaner Production, vol. 277, no. 1, Article ID 123646, 2020.

[29] Y. Fu and J. Zhu, "Operation mechanism for G2B system based on blockchain," Tehnički Glasnik, vol. 26, no. 6, pp. 1841-1852, 2019.

[30] J. Karamachoski, N. Marina, and T. P. Jovan, "Blockchainbased application for certification management," Tehnički Glasnik, vol. 14, no. 4, pp. 488-492, 2020. 